\title{
Control del daño sísmico estructural en pórticos prefabricados de hormigón armado a través de uniones híbridas autocentrantes
}

\author{
Control of structural seismic damage in prefabricated reinforced concrete frames through \\ hybrid-selfcentering connections
}

\section{Juan Marcus ${ }^{1}$ y Rodrigo Thiers ${ }^{2}$}

Fecha de entrega: 23 de enero 2015 Fecha de aceptación: 19 de octubre 2015

\begin{abstract}
${ }^{1}$ Departamento de Ingeniería Civil y Ambiental, Universidad del Bío-Bío, Avda. Collao 1202, Concepción, Chile, jmarcus@jms.cl ${ }^{2}$ Departamento de Obras Civiles, Universidad Técnica Federico Santa María, Avda. España 1680, Valparaíso, Chile, rodrigo.thiers@alumnos.usm.cl
\end{abstract}

La filosofía actual de diseño sísmico de estructuras industriales en Chile, establece como objetivos la protección de vida y la continuidad de operación en la industria. El cumplimiento de estas exigencias permite controlar el daño estructural en base a criterios de resistencia, sin lograr detectar el modo de falla, ni especificar su localización frente a un evento sísmico importante. En el presente trabajo se discute la aplicación de una técnica innovativa de control de daño estructural en pórticos prefabricados de hormigón armado, fundados en suelos granulares, y pertenecientes al proyecto Forestal y Papelera Concepción. Se trata de incorporar uniones hibridas postensadas en las columnas prefabricadas del proyecto, de manera de controlar la disipación de energía en la unión y mantener la rigidez inicial del sistema. Mediante un análisis dinámico no lineal usando el programa Ruaumoko 2D, se compara el posible desempeño del diseño tradicional versus el diseño innovativo. Estos análisis se ejecutan para diversos registros sísmicos chilenos representativos de distintos tipos de suelos. Los resultados del análisis indican que la estructura con el diseño tradicional podría sufrir desplazamientos en el techo del orden de $40 \mathrm{~cm}$, incursionando fuertemente en el rango inelástico, con deformaciones remanentes y concentrando el daño por generación de rótulas plásticas en los extremos de las columnas y algunas vigas no diseñadas para ductilidad. En contraparte, la utilización de uniones híbridas autocentrantes provoca que la estructura recupere su posición original, sin la presencia de deformaciones remanentes.

Palabras clave: hormigón prefabricado, uniones híbridas postensadas, daño sísmico
Current seismic design philosophy of industrial structures in Chile, aims at the protection of life and continuity of operation in the industry. The compliance with these requirements allows controlling the structural damage based on strength criteria, unable to detect the failure mode and specify its location facing a major seismic event. In this paper we discuss the application of an innovative technique to control the structural damage in prefabricated reinforced concrete frames, founded on granular soils, applied in the construction of the Forest and Paper Pulp Plant Concepción. This is done by incorporating hybrid post-tensioned joints in precast columns of the project, this approach aims to control energy dissipation in the union and maintain the initial stiffness of the system. Using a nonlinear dynamic analysis with $2 D$ Ruaumoko software, potential performance in traditional design versus innovative design is compared. The analysis is performed for various Chilean representative seismic records and different types of soils. The results indicate that the structure with the traditional design could suffer displacement in the roof of the order of $40 \mathrm{~cm}$, moving heavily into the inelastic range, with residual deformations, concentrating the damage generation of plastic hinges at the ends of the columns and some beams not designed for ductility. In contrast, the use of hybrid connections causes self-centering effect to the structure returning to its original position, without the presence of remnants deformations.

Keywords: precast concrete, hybrid post-tensioned joints, seismic damage

\section{Introducción}

La filosofía vigente para el diseño sísmico de estructuras industriales en Chile, establece como objetivos la protección de vida y la continuidad de operación en la industria. El 
cumplimiento de estas exigencias no garantiza la ausencia de daño estructural frente a un evento sísmico importante. Este escenario ha generado disputas legales entre inversionistas, empresas constructoras y consultoras de ingeniería estructural, debido a las diferentes expectativas que tienen en términos de desempeño y daño estructural.

Dentro de las tipologías constructivas más utilizadas en estructuras industriales, se destacan los pórticos prefabricados de hormigón armado. Su rapidez de montaje, costos competitivos, excelente resistencia al fuego, y versatilidad arquitectónica, la han erigido como una alternativa válida en el contexto antes mencionado.

No obstante lo anterior, diversas investigaciones teóricas y de terreno, han demostrado que este tipo de estructuras pueden quedar con daños y deformaciones remanentes, luego de eventos sísmicos importantes. Esta situación se evidencia aún más, cuando estas estructuras son fundadas en suelos sueltos o blandos, aumentando la probabilidad de tener que incurrir en disputas legales y gastos adicionales de reparación y rehabilitación estructural.

En la Figura 1, se muestran algunos ejemplos de los daños sísmicos típicos en estructuras prefabricadas de hormigón armado, reportados en Turquía (Saatcioglu et al., 2001; Posada y Wood, 2002; Sezen et al., 2006; Arslan et al., 2006). Los casos antes mencionados se complementan con los daños reportados en China (Zhao et al., 2009), y Nueva Zelanda (Kam et al., 2010, 2011) en tipologías estructurales similares.

Como se puede ver en la Figura 1, los daños típicos en estas estructuras se concentran en las bases de las columnas prefabricadas, debido a la fuerte demanda inelástica a la sección transversal. Estos daños son bastante indeseables, pues impiden la continuidad de operación de la industria e incluso pueden llevar al colapso de la estructura.

Con la evidencia antes mencionada, en los últimos 15 años se han llevado a cabo una serie de iniciativas, tendientes a generar técnicas de diseño para controlar el daño en estructuras prefabricadas de hormigón armado (Pampanin, 2005). Una de las primeras iniciativas, fue un sistema de uniones secas dúctiles denominado U.S PRESS (PREcast Seismic Structural System), ensayado en la Universidad de California (Priestley, 1991, 1996; Priestley et al., 1999), con cables postensados desprovistos de adherencia. La
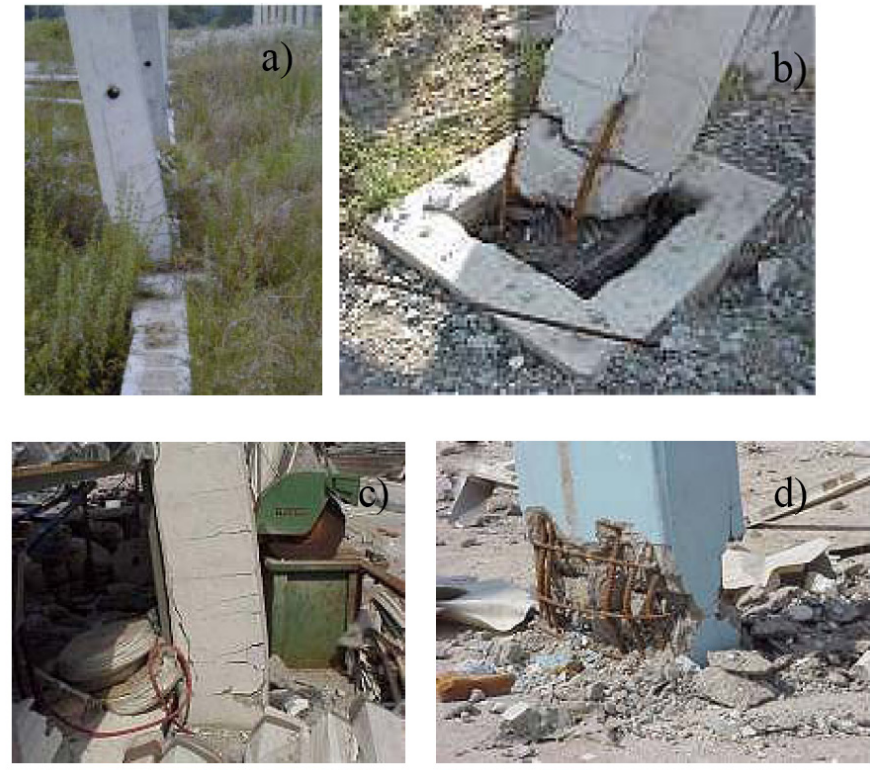

Figura 1: Patrones de daños sísmicos presentes en columnas prefabricadas industriales de hormigón armado en Turquía; a) daño concentrado por rótula flexural en base de columna (Posada y Wood, 2002), b) daño concentrado en base de columna con fundación del tipo zócalo, c) daño por rótula flexural distribuida en $800 \mathrm{~mm}$ de altura de columna y d) daño severo por rótula flexural concentrada en base de columna (Saatcioglu et al., 2001)

demanda inelástica se acomoda dentro de la conexión, a través de la abertura y cierre de un espacio existente, en un movimiento del tipo balanceo. Como resultado se logró una configuración que puede desarrollar desplazamientos inelásticos, limitando el daño estructural y asegurando una capacidad auto-centrante completa. El comportamiento histerético de la unión es esencialmente no lineal-elástico, con una reducida capacidad de disipación de energía.

Como mejora al sistema PRESS, Stanton et al. (1997) desarrollaron el concepto de uniones híbridas, donde combinaron las propiedades de auto-centrado y disipación de energía, a partir del uso de tendones post-tensados sin adherencia y barras longitudinales de acero dúctil no pretensadas. Bajo acciones sísmicas de intensidad moderada, el clásico mecanismo de rótula plástica, es reemplazado por un mecanismo de balanceo controlado en la interfaz crítica, sin daño en el elemento estructural. Mientras los tendones post-tensados proveen acciones restitutivas auto-centrantes, las barras de acero dúctiles actúan como disipadores de energía absorbiendo el impacto de las cargas sísmicas. Adicionalmente, para prevenir fracturas prematuras de estas barras de acero dúctiles, se les otorga una pequeña longitud sin adherencia en las 
cercanías de la unión híbrida, a través de una vaina.

Este particular mecanismo auto-centrante-disipador de las uniones híbridas, es generalmente descrito por un comportamiento histerético con forma de bandera. En la Figura 2 se esquematiza el principio de funcionamiento de las uniones híbridas auto-centrantes.

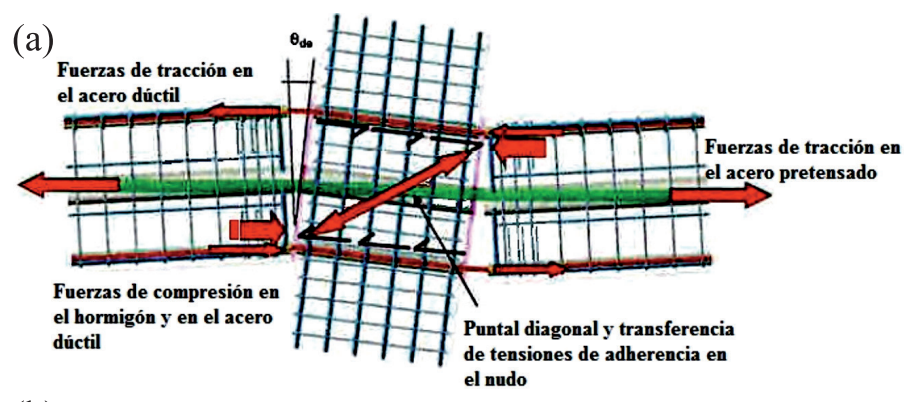

(b)

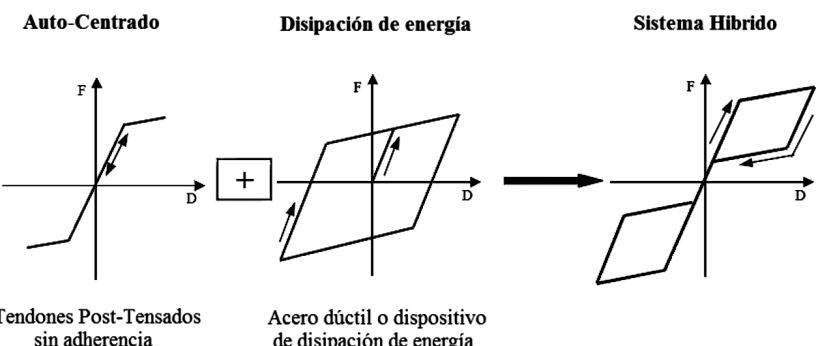

Figura 2: Esquemas de funcionamiento de uniones híbridas auto-centrantes. a) Esquema de fuerzas internas en unión vigacolumna y b) modelos constitutivos con ciclos histeréticos con forma de bandera (Buchanan et al., 2011)

En este trabajo se ilustra la incorporación del sistema de uniones híbridas auto-centrantes a un proyecto industrial de pórticos prefabricados de hormigón armado. Las estructuras fueron fundadas sobre estratos profundos de suelos arenosos sueltos en la comuna de Coronel, Chile. A través de análisis numéricos no lineales, se compara la respuesta de la estructura con y sin uniones híbridas, frente a acelerogramas sísmicos chilenos. Finalmente, se comentan los detalles de construcción de la solución, sus posibles mecanismos de falla y las eventuales ventajas comparativas en términos de control de daño. De esta forma, se pretende contribuir a la difusión de este sistema innovativo en zonas de alta sismicidad.

\section{Materiales y métodos}

\section{Caracterización del proyecto de estudio con uniones híbridas}

El proyecto consiste en una nave industrial de $1920 \mathrm{~m}^{2}$, conformada por pórticos de hormigón prefabricado posttensado, con altura máxima de columnas de $18.5 \mathrm{~m}$, emplazada en una zona costera de alta sismicidad y fundada en un suelo arenoso suelto con una densidad relativa de $50 \%$ en promedio. Esta nave se denomina M3A, y en la Figura 3 se muestran las vistas en planta y transversal del proyecto.

(a)

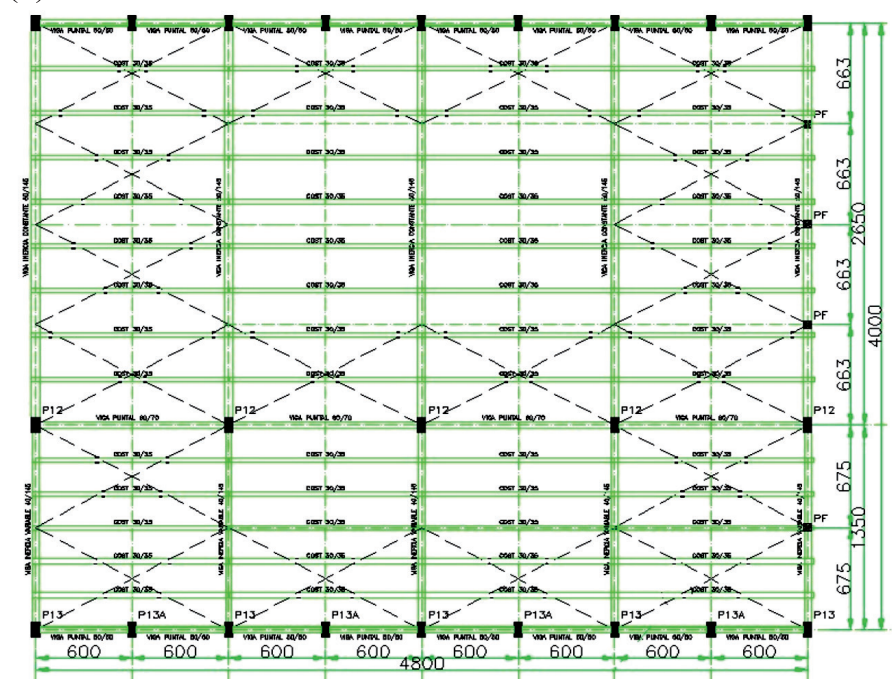

(b)

(D)

(A)

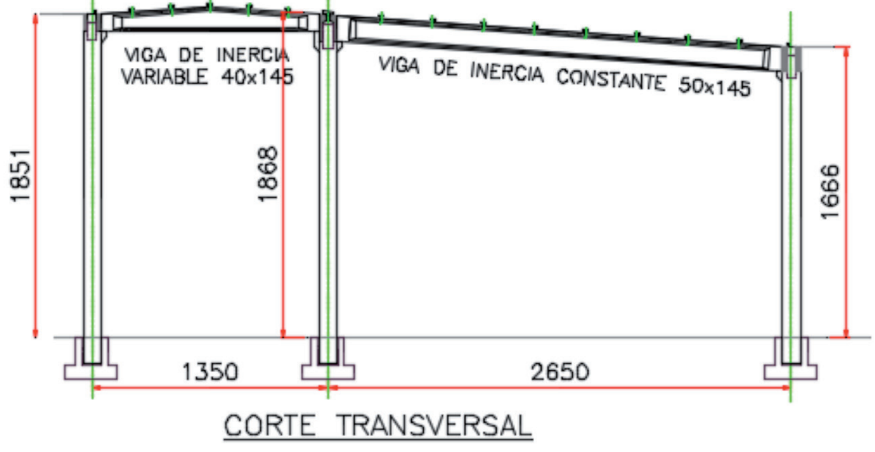

Figura 3: Esquemas en a) planta y b) elevación del proyecto (dimensiones en $\mathrm{cm}$ )

Originalmente, el proyecto contemplaba un sistema de uniones rígidas hormigonadas en terreno (uniones húmedas), con pilares y vigas postensadas, de dimensiones que variaban desde los $40 \mathrm{~cm}$ de ancho, hasta $145 \mathrm{~cm}$ de alto. Sin embargo, la revisión del diseño sísmico sugirió la inclusión de uniones híbridas en los extremos de los pilares, de manera de minimizar el daño estructural y evitar fallas posiblemente frágiles en los nudos más allá de las uniones híbridas, que siguen siendo rígidas y hormigonadas en terreno. En la Figura 4 se muestran algunos detalles constructivos de los pilares y sus uniones híbridas. 
(a)

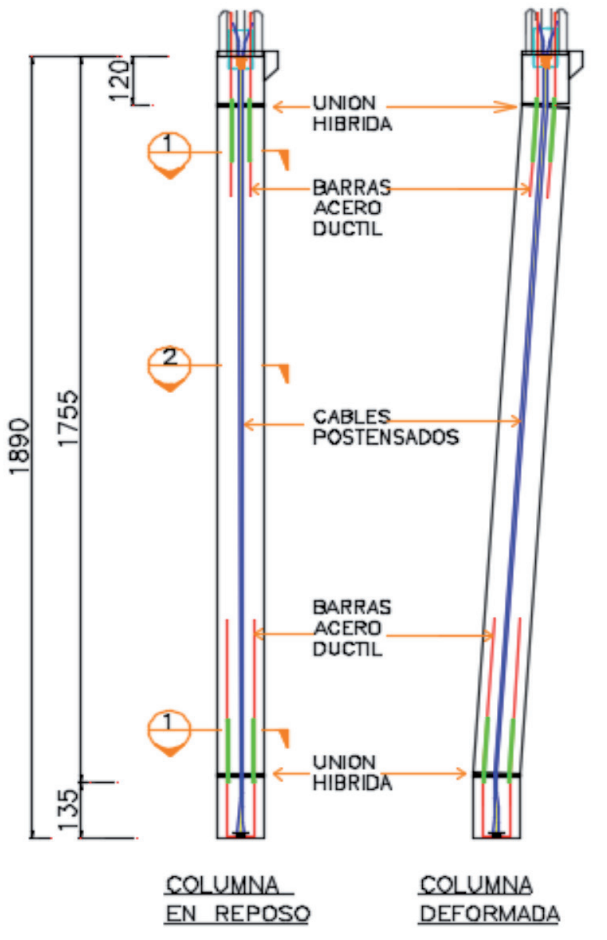

(b)
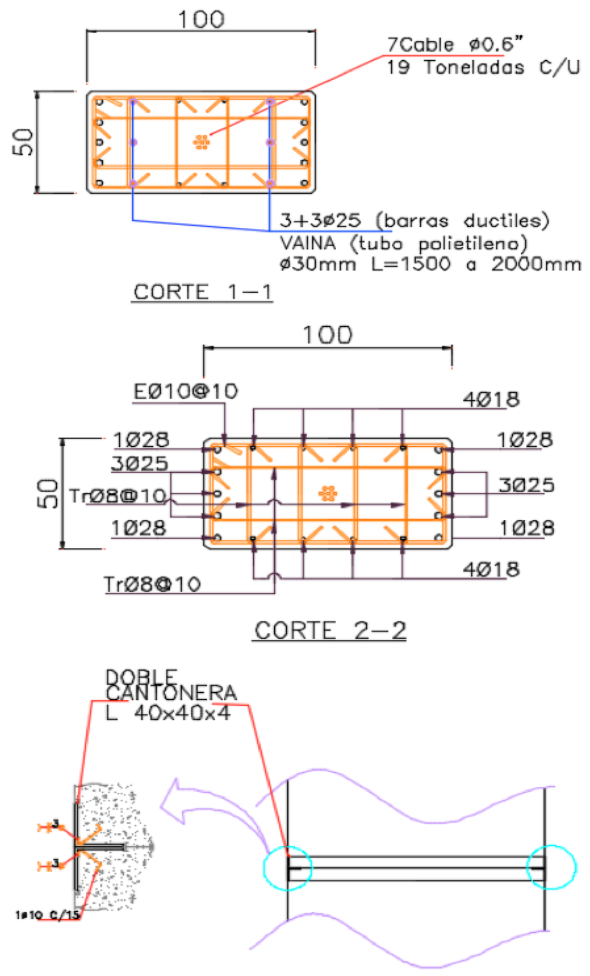

DETALLE UNION HIBRIDA

Figura 4: Detalles constructivos de columnas con uniones híbridas, a) columna eje E y esquema cinemática y b) cortes y detalles transversales

Por otro lado, en la Figura 5 se muestran algunas imágenes del proyecto en etapa de construcción.
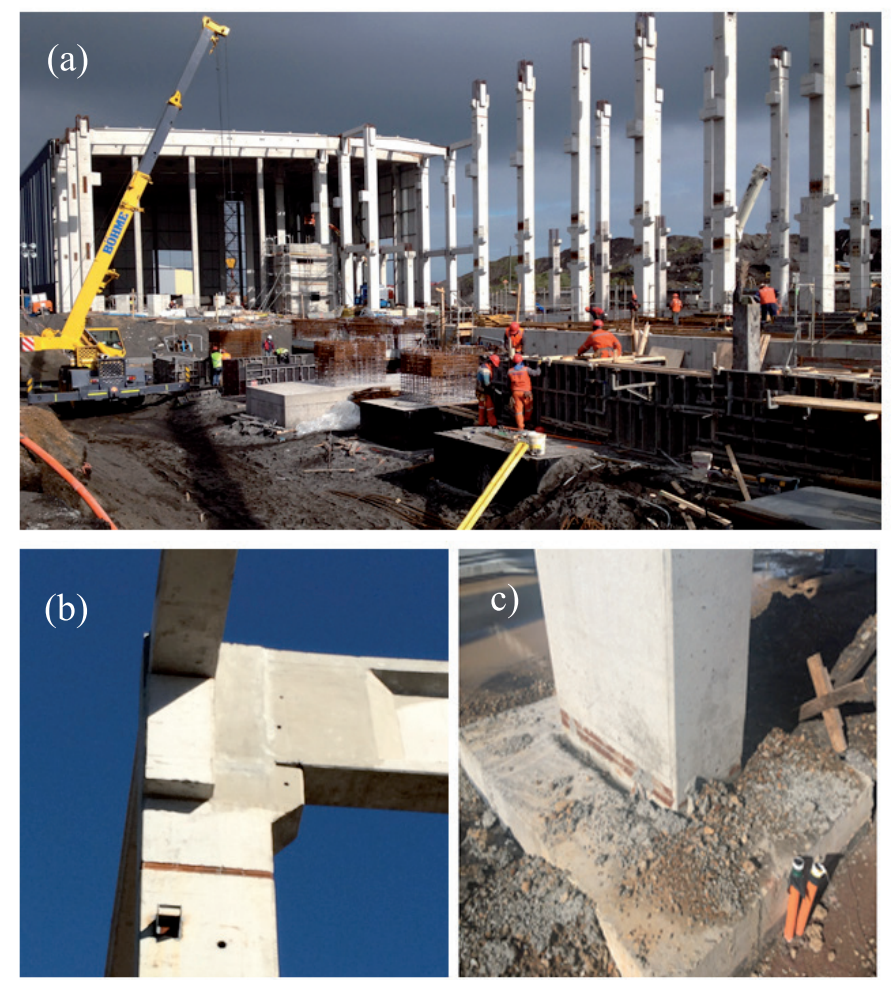

Figura 5: (a) Construcción y montaje de elementos estructurales, (b) unión híbrida en borde superior y c) inferior de una columna

\section{Análisis de la estructura con uniones híbridas}

Para evaluar el nivel de daño del proyecto con y sin uniones híbridas, se realizaron simulaciones numéricas no lineales de los pórticos. Los análisis se hicieron con el programa Ruaumoko 2D (Carr, 2004). En la Figura 6, complementada con la Tabla 1, se muestra uno de los modelos de análisis, con sus respectivas secciones críticas, localizadas en las uniones postensadas en las columnas.

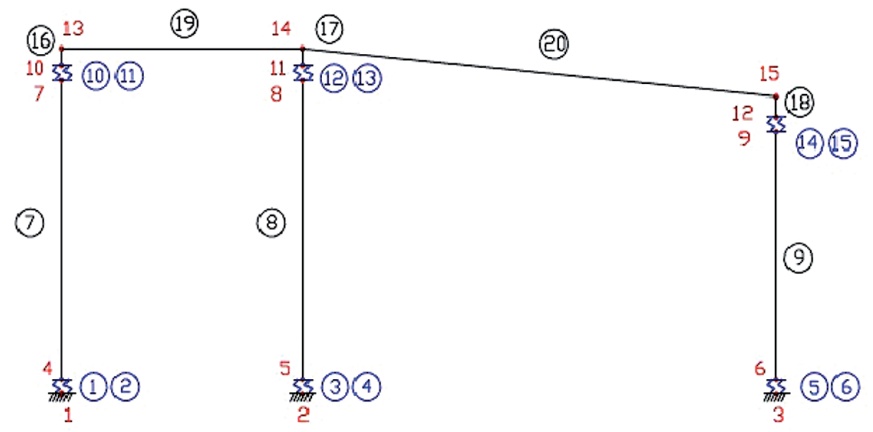

Figura 6: Modelo de análisis del marco transversal típico 
Tabla 1: Nomenclatura de elementos utilizados en el modelo mostrado en Figura 6

\begin{tabular}{|l|l|}
\hline Elementos & Descripción \\
\hline 1-3-5-10-12-14 & $\begin{array}{l}\text { Resorte rotacional: 7 cables 0.6” pos- } \\
\text { tensados (bilineal elástica) }\end{array}$ \\
\hline $2-4-6-11-13-15$ & $\begin{array}{l}\text { Resorte rotacional: acero especial dúctil } \\
\text { disipador 3+3 } \$ 25 \text { (bilineal inelástica) }\end{array}$ \\
\hline $7-8-9-16-17-18$ & Columna 50x100cm (lineal elástica) \\
\hline 19 & Viga inercia variable (lineal elástica) \\
\hline 20 & Viga inercia constante (lineal elástica) \\
\hline
\end{tabular}

Los cables postensados y las barras de acero dúctil (disipadores) de la unión híbrida, se incluyeron en el análisis con resortes rotacionales en paralelo, siguiendo las leyes histeréticas y los modelos físico-conceptuales mostrados en la Figura 7.

Las leyes histeréticas indicadas en la Figura 7, corresponden a una ley bilineal inelástica para el acero dúctil y una ley bilineal elástica para los cables postensados. En la Tabla 2 , se muestran los valores de los parámetros utilizados, donde $k_{\mathrm{o}}$ es la rigidez rotacional elástica, $r$ es la razón entre la rigidez rotacional post-fluencia y la rigidez rotacional elástica y $M_{\mathrm{y}}$ es el momento flector de fluencia. Para su determinación, se siguieron las recomendaciones propuestas por Celik y Sritharan (2004).

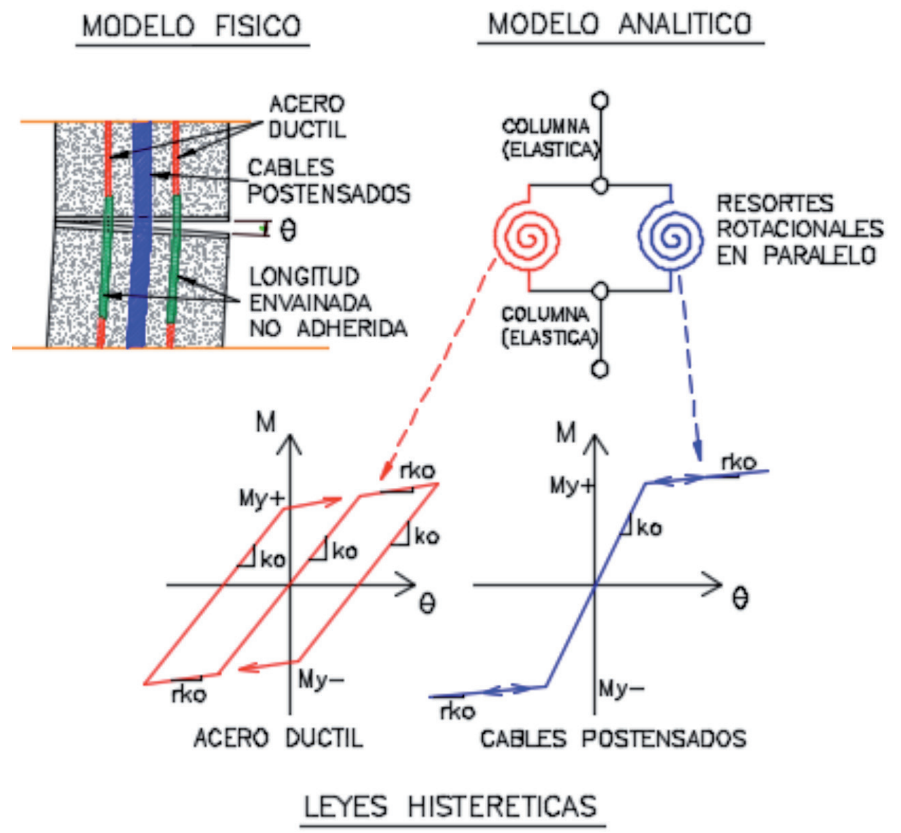

Figura 7: Modelo físico, analítico y leyes histeréticas de barras de acero especial dúctil (disipadores) y cables postensados
Tabla 2: Parámetros utilizados en leyes histeréticas

\begin{tabular}{|l|c|c|c|}
\hline & $\begin{array}{c}k_{\mathrm{o}} \\
\mathrm{Tm} / \mathrm{rad}\end{array}$ & $r$ & $\begin{array}{c}M_{\mathrm{y}} \\
\mathrm{Tm}\end{array}$ \\
\hline Acero dúctil & 37110 & 0.015 & 37.11 \\
\hline Cables postensados & 74096 & 0.004 & 74.10 \\
\hline
\end{tabular}

Como evaluación preliminar de la estrategia de modelación, se realizó un análisis incremental en dos sentidos (pushpull), aplicando una historia de desplazamientos en el techo, tal como indica la Figura 8.

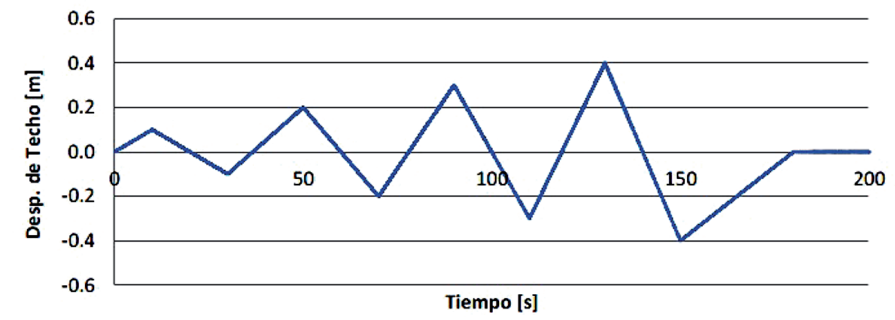

Figura 8: Historia de desplazamientos aplicada a modelo preliminar

El resultado preliminar mostrado en Figura 9, confirma que el modelo es capaz de representar la respuesta de tipo bandera, que caracteriza a estas uniones. De esta forma, es posible realizar análisis tiempo-historia más detallados, utilizando acelerogramas que se consideran representativos de las condiciones locales sísmicas.

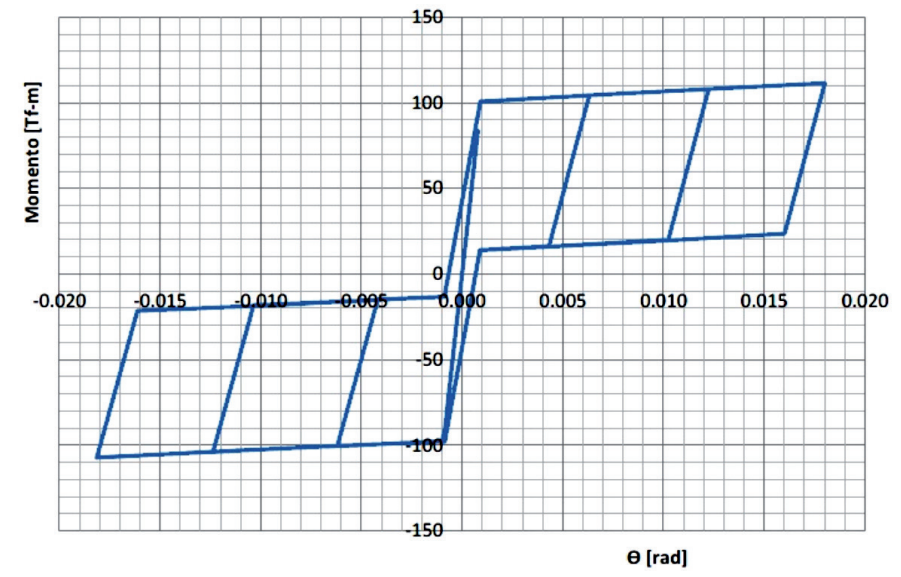

Figura 9: Diagrama momento-rotación obtenido en una de las uniones híbridas del borde inferior de las columnas 


\section{Análisis de la estructura original sin uniones híbridas}

Para poder comparar el daño estructural y evaluar la efectividad de la solución propuesta, también es necesario modelar y analizar la estructura original sin uniones híbridas. Esto se realizó con el programa Ruaumoko 2D, a través de un análisis dinámico no lineal (Thiers, 2014). El modelo del marco transversal de la nave es similar al de la Figura 6, pero sin uniones híbridas. De esta forma, se consideraron las tres columnas, la viga de inercia variable y la viga de inercia constante.

Para modelar la no linealidad de la estructura se asume que las secciones críticas están en los extremos de los elementos y que el nudo tiene la resistencia suficiente para permitir que las secciones críticas fluyan antes de que el nudo falle. Para las vigas y las columnas se utilizaron elementos de plasticidad concentrada en sus extremos, del tipo Giberson (Sharpe, 1974), donde las rotaciones se concentran en los extremos del elemento. En la Figura 10 se muestra el esquema conceptual de este tipo de elementos.

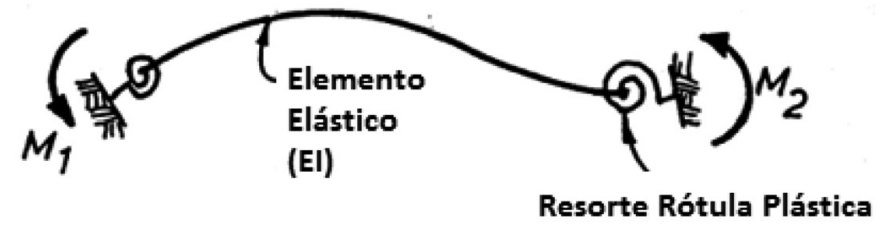

Figura 10: Elemento tipo Giberson para modelar no linealidad en estructura original sin uniones híbridas

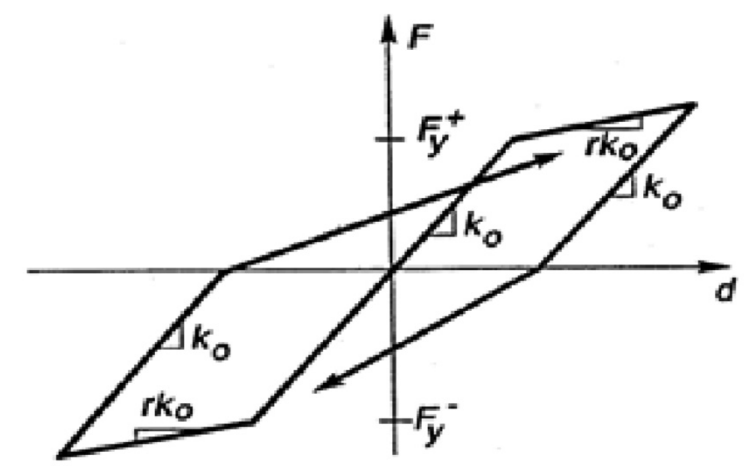

Figura 11: Ley de histéresis de Clough para modelar no linealidad en elementos de hormigón armado (caso original sin uniones híbridas)

La rigidez del resorte rotacional indicado en la Figura 10, se determina a partir de la rigidez tangente, correspondiente al nivel de deformación de la ley de histéresis considerada.
En este caso se utilizó la ley de histéresis de Clough (1966), mostrada en la Figura 11, que corresponde a la ley de Takeda modificada, con parámetros $\alpha$ y $\beta$ igual a cero. Como los elementos de hormigón armado tienen baja rigidez post fluencia, se utilizó un valor de $r$ igual a $1 \%$.

\section{Resultados y discusión}

El análisis no lineal se hizo con el registro sísmico obtenido en Concepción 2010, mostrado en la Figura 12 , en condiciones locales de sismicidad y tipo de suelo similares al lugar donde está el proyecto. Ambos suelos corresponden a arenas Bío Bío, sueltas en superficie con densidades relativas del orden de 50\%. Además, la distancia entre la estructura del proyecto y la estación acelerográfica es de aproximadamente $20 \mathrm{~km}$.

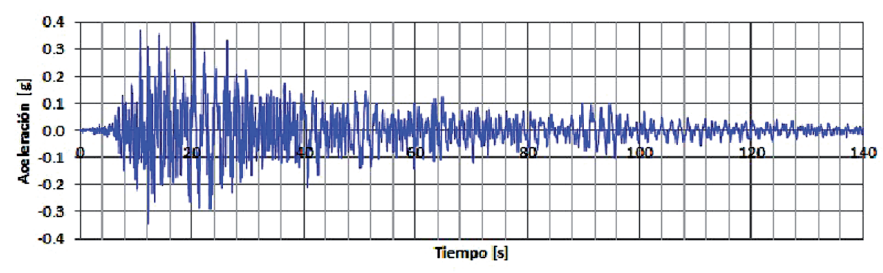

Figura 12: Acelerograma estación Concepción centro, 27 febrero 2010 , orientación $60^{\circ}$ con respecto al norte (Boroschek et al., 2010)

\section{Modelo sin uniones híbridas}

Como primera etapa se realizó un análisis de la estructura original sin uniones híbridas. En la Figura 13 se muestra la respuesta de desplazamiento lateral en el techo calculado para el registro mostrado en Figura 12.

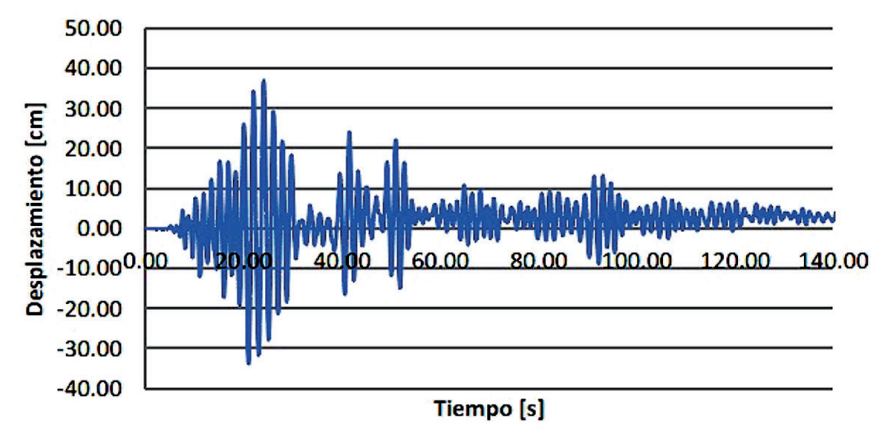

Figura 13: Historia de desplazamiento lateral en el techo (sin uniones híbridas)

Como se puede ver en la Figura 13, los desplazamientos en el techo del pórtico sin uniones híbridas alcanzan los 
$37 \mathrm{~cm}$. Este nivel de desplazamientos es importante, ya que está asociado a la presencia de daños en las uniones y posibles fallas frágiles no localizadas por el método de diseño en base a fuerzas. Además, se deben tomar precauciones en los elementos que se unen a la nave en la dirección transversal, para poder sostener este nivel de deformaciones. De manera complementaria, se observa la presencia de desplazamientos remanentes, del orden de los $3 \mathrm{~cm}$. En la Figura 14 se muestra el diagrama momentocurvatura obtenido en una de las bases de los pilares prefabricados.

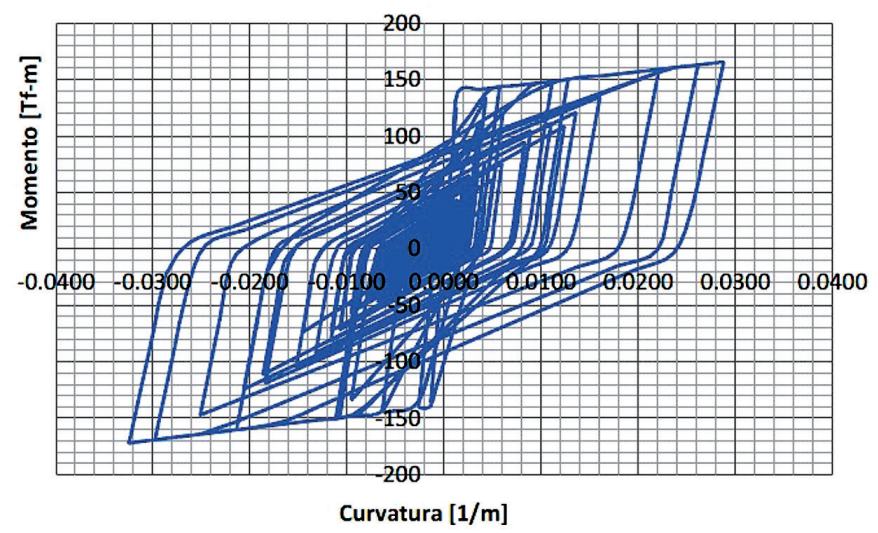

Figura 14: Diagrama momento-curvatura para columna con uniones híbridas (extremo inferior)

Los resultados observados en la Figura 14 muestran una fuerte incursión en el rango no lineal, con daños importantes en los pilares prefabricados sin uniones híbridas. Las máximas curvaturas alcanzadas fueron del orden de 0.0324 $\mathrm{m}^{-1}$, por lo tanto, se debe confinar adecuadamente, dotar de ductilidad y reforzar los nudos.

En la Figura 15 se muestran las secciones más solicitadas y un posible mecanismo de colapso del pórtico. A partir de estos resultados se recomienda proveer confinamiento en los nudos y en las secciones de los elementos donde se espera que se produzcan rótulas plásticas. Además se debe detallar la enfierradura para evitar fallas frágiles e intentar localizar el daño en una sección crítica preestablecida. Estas recomendaciones son muy difíciles de lograr con el tipo de solución constructiva original. Los análisis de un diseño tradicional indican que se espera daño en las secciones críticas, con alargamientos unitarios en el acero de hasta un $4 \%$.

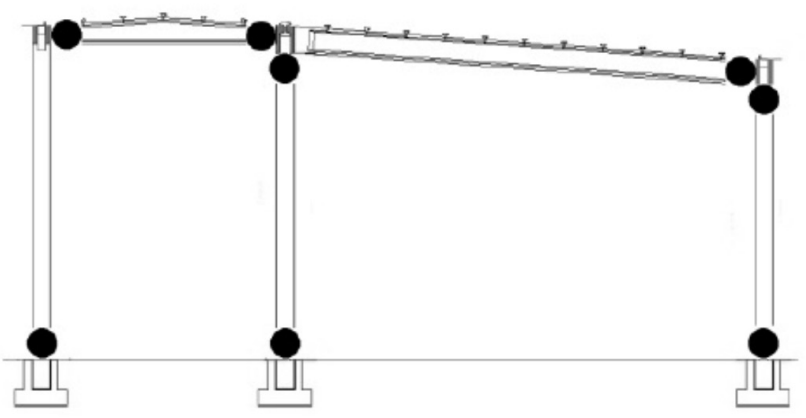

Figura 15: Posible mecanismo de colapso en el pórtico diseñado con métodos tradicionales

\section{Modelo con uniones híbridas}

El tipo de daño esperado sugiere la necesidad de controlar sus efectos y limitar los desplazamientos remanentes en la estructura. De esta forma, un sistema de uniones híbridas en los pilares prefabricados surge como una estrategia adecuada para lograr estos objetivos. Lo anterior se debe a la posibilidad de este sistema de concentrar el daño en las aberturas y disipar la energía con las barras desprovistas de adherencia en un tramo, evitando las concentraciones de alargamientos unitarios en el acero. El diseño de estas uniones se hizo de acuerdo a las recomendaciones de ACI T1.2-03 (2003).

En la Figura 16 se muestra el desplazamiento lateral en el techo, obtenido para el modelo con uniones híbridas, sometido al registro sísmico Concepción 2010 longitudinal.

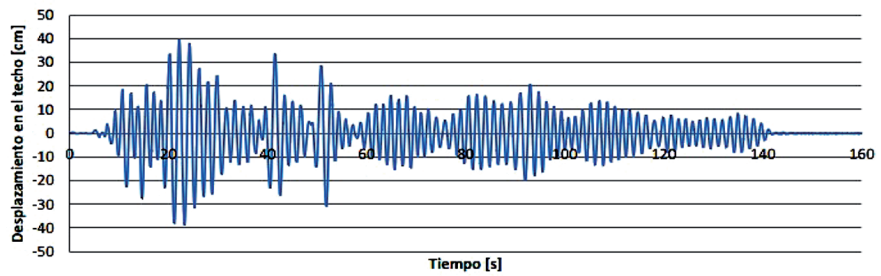

Figura 16: Historia de desplazamiento lateral en el techo para estructura con uniones híbridas

Según lo mostrado en la Figura 16, se observa que la estructura alcanzaría desplazamientos en el techo del orden de $40 \mathrm{~cm}$, levemente mayores que la estructura tradicional. Sin embargo, no habría daño estructural asociado y la estructura recuperaría su posición original sin deformaciones remanentes, reflejando claramente las 
ventajas del sistema propuesto. Esto es posible ya que el resto de los elementos estructurales se diseñaron por capacidad, para asegurar la formación del mecanismo de colapso deseado. De manera complementaria, en las Figuras 17 y 18 se muestran algunos diagramas momentorotación obtenidos en las uniones híbridas de las columnas $\mathrm{y}$ en las vigas, respectivamente.
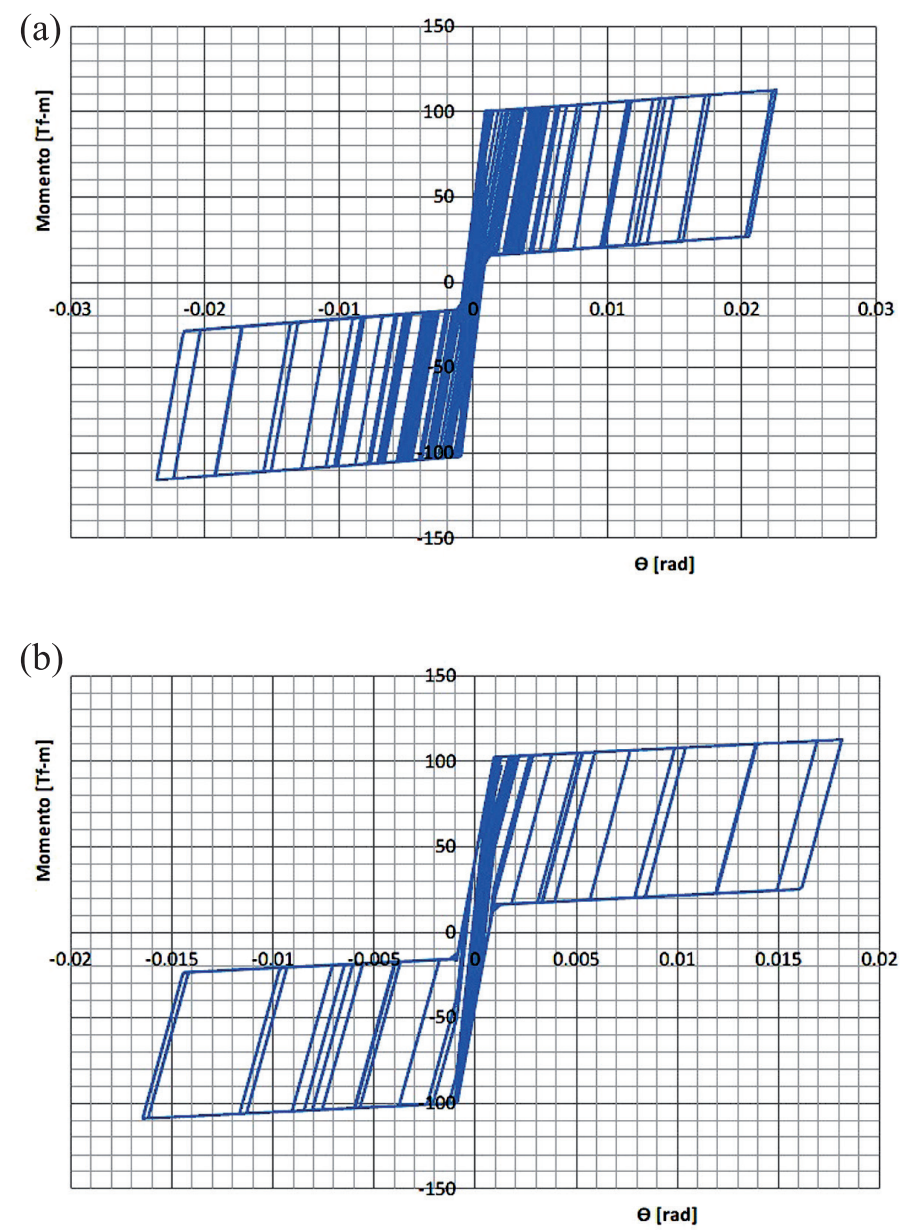

Figura 17: Diagramas momento-rotación para una columna con uniones híbridas, a) extremo inferior y b) extremo superior

De la Figura 18 se desprende que las vigas prefabricadas no incursionan en el rango no lineal, permaneciendo elásticas durante el sismo. Sin embargo deben poseer una resistencia adecuada para que la no linealidad se concentre en la unión híbrida de las columnas. En la Figura 19 se muestra el esquema de resistencia requerida a flexión para que las vigas permanezcan elásticas, en las secciones cercanas a los nudos.

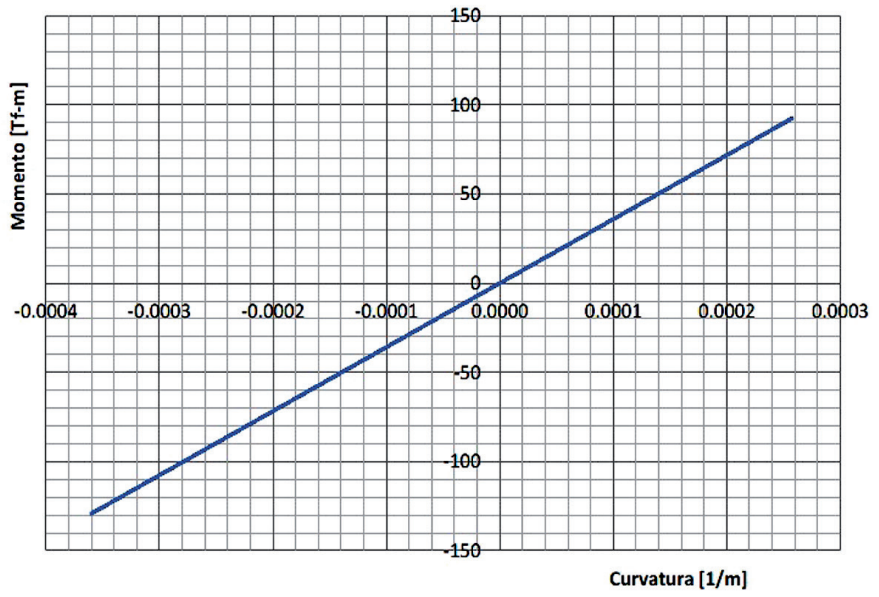

Figura 18: Diagrama momento-curvatura en extremo derecho de viga prefabricada de inercia constante

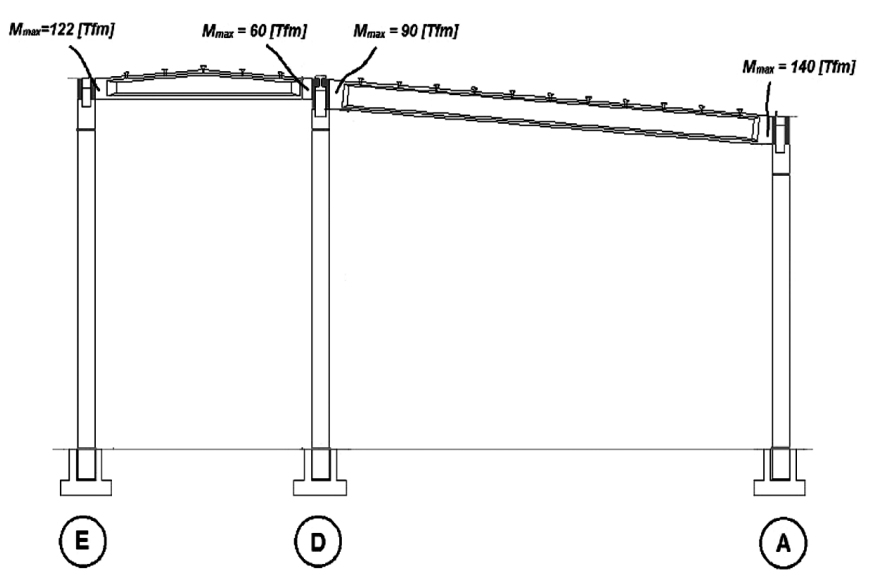

Figura 19: Resistencia requerida de vigas a flexión para permanecer elásticas

\section{Modelo con uniones híbridas para otros registros sísmicos}

Para comparar las demandas de desplazamientos correspondientes a registros en diferentes condiciones de suelo, se obtuvo la respuesta de la estructura modificada con uniones híbridas sometida a registros de Concepción y Viña del Mar. En la Figura 20 se resumen los resultados obtenidos. En azul se dibujó la respuesta momento rotación de la unión en la base de la columna del eje A sometida al registro Concepción centro 2010. Sobre esta curva se marcaron los valores máximos de momento y rotación obtenidos en los análisis realizados con los demás registros. Se puede ver que para los diferentes registros considerados, propios de diversas condiciones del suelo 
bajo la estructura, la demanda de capacidad de deformación es la variable. En todos los casos, a excepción de los registros obtenidos directamente sobre roca, se alcanza la resistencia disponible, mostrando lo evidente, que el diseño sísmico constituye un problema de desplazamientos y no de resistencia.

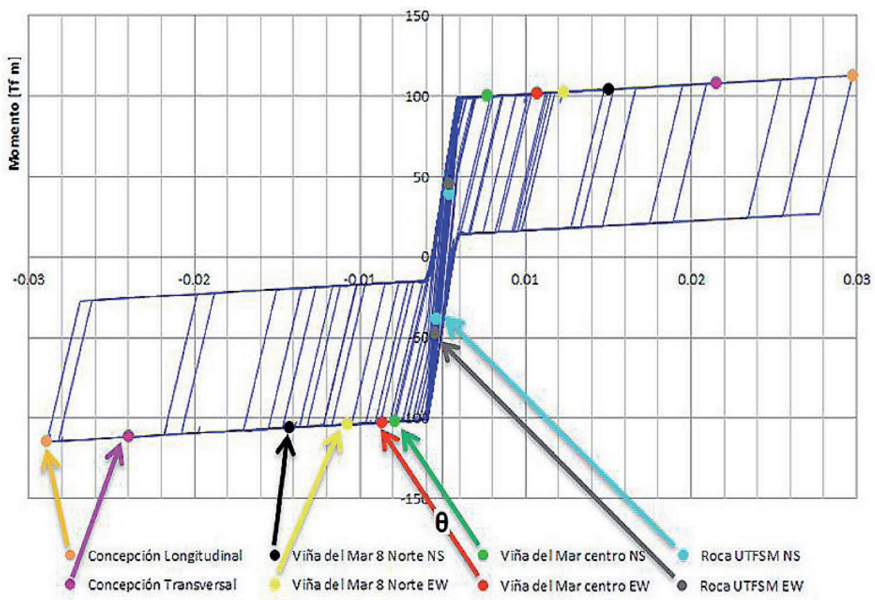

Figura 20: Diagrama momento-rotación de unión híbrida en la base de la columna del eje A para diversos registros sísmicos

\section{Conclusiones}

En este estudio se mostró analíticamente la efectividad de la utilización de uniones híbridas auto centrantes, como medio para controlar el daño sísmico estructural en pórticos prefabricados de hormigón armado. Esta solución constructiva es aún más relevante cuando la estructura se encuentra emplazada en suelos arenosos sueltos con posibilidades de sufrir desplazamientos sísmicos laterales del orden de $40 \mathrm{~cm}$.

Al comparar el comportamiento sísmico del pórtico diseñado tradicionalmente versus el pórtico diseñado con uniones híbridas, se puede destacar que este último permite controlar el mecanismo de colapso, permitiendo que las vigas permanezcan elásticas, concentrando el daño en la abertura de las uniones híbridas de las columnas, disipando esa energía a través de las barras dúctiles y proveyendo un auto-centrado a través de los cables postensados. Estas ventajas comparativas son muy difícil de alcanzar en pórticos diseñados tradicionalmente en base a fuerzas.

Del punto de vista constructivo, se observó que es posible implementar este sistema de uniones híbridas en estructuras industriales reales, las cuales por razones propias de continuidad de operación, requieren minimizar los daños y limitar las deformaciones remanentes frente a eventos sísmicos importantes.

\section{Agradecimientos}

Los autores agradecen la información y el aporte a este artículo entregado por los ingenieros Manuel Robles (encargado del diseño de la estructura prefabricada) y Patricio Bonelli (revisor sísmico estructural del proyecto, quien sugirió y asesoró el diseño de las uniones híbridas). Además se agradece la colaboración del Ing. Alexander Opazo, docente de la Universidad del Bío Bío quien colaboró en la redacción de este documento y de las empresas constructoras Prefabricados Estructurales S.A (fabricación de elementos prefabricados) y CME (montaje en terreno), por sus importantes gestiones y acompañamiento en las visitas a obra.

\section{Referencias}

ACI T1.2-03 (2003). Special hybrid moment frames composed of discretely jointed precast and post-tensioned concrete members. American Concrete Institute and Innovation Task Group 1 and collaborators, Farmington Hills, MI

Arslan, M.H., Korkmaz, H.H. and Gulay, F.G. (2006). Damage and failure pattern of prefabricated structures after major earthquakes in Turkey and shortfalls of the Turkish Earthquake code. Engineering Failure Analysis 13(4), 537-557

Boroschek, R., Soto, P. y Leon, R. (2010). Registros del terremoto del Maule $\mathrm{Mw}=8.8,27$ de febrero de 2010. Red Nacional de Acelerógrafos, Universidad de Chile, Informe RENADIC 10(05)

Buchanan, A.H., Bull, D., Dhakal, R.P., MacRae, G., Palermo, A. and Pampanin, S. (2011). Base isolation and damage-resistant technologies for improved seismic performance of buildings. Royal Commission of Inquiry into Building Failure Caused by the Canterbury Earthquakes. University of Canterbury

Carr, A.J. (2004). Ruaumoko 2D-Inelastic dynamic analysis. Department of Civil Engineering, University of Canterbury, Christchurch

Celik, O. and Sritharan S. (2004). An evaluation of seismic design guidelines proposed for precast concrete hybrid frame systems. Iowa State University 
Clough, R.W. (1966). Effect of stiffness degradation on earthquake ductility requirements. Internal Report, University of California, Berkeley

Kam, W.Y., Pampanin, S., Dhakal, R.P., Gavin, H., and Roeder, C.W. (2010). Seismic performance of reinforced concrete buildings in the September 2010 Darfield (Canterbury) earthquakes. Bulletin of the New Zealand Society of Earthquake Engineering 43(4), 340-350

Kam, W.Y., Pampanin, S. and Elwood, K. (2011). Seismic performance of reinforced concrete buildings in the 22 February Christchurch (Lyttleton) earthquake. Bulletin of the New Zealand Society of Earthquake Engineering 44(4), 239-279

Pampanin, S. (2005). Emerging solutions for high seismic performance of precast/prestressed concrete buildings. Journal of Advanced Concrete Technology 3(2), 207-223

Posada, M. and Wood, S. (2002). Seismic performance of precast industrial buildings in Turkey. In 7th US National Conference on Earthquake Engineering (7NCEE)

Priestley, M.J.N. (1991). Overview of the PRESSS research programme. PCI Journal 36(4), 50-57

Priestley, M.N. (1996). The PRESSS program: current status and proposed plans for phase III. PCI Journal 41(2), 22-40

Priestley, M.J.N., Sritharan, S., Conley, J.R. and Pampanin, S. (1999). Preliminary results and conclusions from the PRESSS five-story precast concrete test building. PCI Journal 44(6), 4267
Saatcioglu, M., Mitchell, D., Tinawi, R., Gardner, N. J., Gillies, A. G., Ghobarah, A. and Lau, D. (2001). The August 17, 1999, Kocaeli (Turkey) earthquake damage to structures. Canadian Journal of Civil Engineering 28(4), 715-737

Sezen, H. and Whittaker, A.S. (2006). Seismic performance of industrial facilities affected by the 1999 Turkey earthquake. Journal of Performance of Constructed Facilities 20(1), 28-36

Sharpe, R. (1974). The seismic response of inelastic structures. $\mathrm{PhD}$ thesis, Department of Civil Engineering, University of Canterbury

Stanton, J., Stone, W.C. and Cheok, G.S. (1997). A hybrid reinforced precast frame for seismic regions. PCI Journal 42(2), 20-32

Thiers, R. (2014). Daños en edificios de hormigón armado y su relación con el suelo - Terremoto de Chile 2010. Tesis de Magister, Universidad Técnica Federico Santa Maria

Zhao, B., Taucer, F. and Rossetto, T. (2009). Field investigation on the performance of building structures during the 12 May 2008 Wenchuan earthquake in China. Engineering Structures 31(8), 1707-1723 